\title{
Competing Histories of Technology: Recognizing the Vital Role of International Scientific Communities behind the Innovation of the Internet
}

\author{
Christopher Leslie \\ Polytechnic Institute of New York University, Brooklyn NY, USA \\ cleslieapoly.edu
}

\begin{abstract}
One way to make the history of computing relevant is to explain how different histories are in competition with each other and how they support quite different technology policy. The prevalent history that the US military created the Internet hides the international spirit of goodwill and cooperation that made a particular implementation of Internet happen. This is not just an academic issue. The success of the Internet encourages us to ask how we can continue to innovate the technologies of the Internet or new technologies of a similar power. The myth that the military created the Internet also supports the idea that it was designed on purpose according to a plan. This could not be further from the truth. In fact, the most interesting thing about the Internet is that, in its history, it had a tendency to violate the plans set forth. The international community invigorated the kind of inquiry that would lead to the Internet, not the United States in isolation. In order to create something like the Internet, then, we need to provide an environment for international exchange and cooperation, not maintain proprietary secrets and work in a disciplined environment of practical research.
\end{abstract}

Keywords: History of the Internet, packet switching, technology policy, innovation.

\section{Introduction}

The facts seem simple: The United States military funded a project to build, test, and improve a packet-switched network in the 1970s that provided the groundwork upon which the modern Internet was built. This story has been told many times, but the facts are not the most interesting thing in the discipline of history; to the historian, the implication of these facts is more important. Is this a story of military contracts providing technology to civilians? Is this a story of American ingenuity and technological superiority? Or is it a story of how academic researchers and commercial enterprises made a technology by innovating science developed for the military? One way to make the history of computing relevant is to explain how different histories are in competition with each other and how they support quite different technology policies. Because the Internet has been so successful, it is 
important to tell the right history of its development so that future innovation can be supported. Unfortunately, the story of the Internet is frequently told in such a way that hides the vital role of the international scientific community.

Historians of technology frequently rail against "whiggish" histories, or histories that interpret the past by explaining how we arrived at the present, as if there were a clear, linear path from an identifiable origin to the modern day. The history of the Internet is sometimes referred to in such a manner. Where Wizards Stay Up Late [1] is a perfect example. This book combines interviews with many people who helped manage projects that built up to the Internet, and the authors provide an indispensable resource for someone who wants to know about the Internet's history. Nerds: 2.0.1: A Brief History of the Internet similarly relates a tale for dramatic effect of the slow building of technology until the present day [2]. Both of these texts are useful, but an unintended consequence of their approach is that it seems as if technological devices grow with their own inertia, one development adding itself to the next until the perfected whole is achieved. Shorthanded accounts of the history of the Internet proliferate in newspapers and college history and media studies courses, promoting the idea that the Internet grew linearly and inevitably from a simple idea into a complex version in the present. An historian of technology would protest that these versions of the story make the development seem inevitable and free from connection to the economic, legal, political, social, ideological circumstances, let alone from dependence on existing technology. This might suggest that there is only one best way to make a worldwide computer network or, by extension, any other technological innovation. Although this approach is good for television series and mass-market books, it is not suitable for those interested in technological innovation. Given the success of the Internet, engineers and computer scientists interested in inventing in a similar environment need a more nuanced history.

Teleological approaches have been shunned for twenty years, and historians have begun offered alternatives to this approach. Janet Abbate's Inventing the Internet [3] was one of the first, telling as it did the ways in which Internet history was woven together with a larger institutional framework and existing technological achievements. Milton Mueller's Ruling the Root [4] adds counterfactual histories, hypothesizing for readers how things could have worked differently, to his discussion of how a new form of internet governance emerged at the end of the 1990s. Martin Campbell-Kelly and Daniel D. Garcia's recent article "The History of the Internet: The Missing Narratives" similarly makes hypothetical forays into what could have brought together widespread computer networks besides TCP/IP and examines the consequences and opportunities of each; it is not as if the Internet grew from the ARPANet "as a tree from a tiny acorn" [5]. As well, Byung-Keun Kim [6] makes an effort to describe the multiple histories of networks that led to the Internet, and Luis Arroyo has documented a 1972 commercial packet-switched network designed independently in Spain [7]. At this conference, we have heard more about local histories of computers that are evidence of large community of professionals engaged in innovating computers and networks. The history of this community, with its multiple efforts to advance computing technology, needs to be elaborated because it helps to explain why the growth of the Internet was not inevitable, but one that relied on a particular ethic of cosmopolitan cooperation. This version of the history of the Internet is more appropriate for engineers and computer scientists who hope to 
innovate new technology because it helps them to draw connections between the technological, political, and economic framework that enabled certain aspects of Internet innovation while at the same time forestalling other possibilities.

In this paper, I would like to build on these studies by employing a notion of the user from Science and Technology Studies. As pointed out by the contributors to the volume How Users Matter [8], it is difficult and even inappropriate to separate a technical, design stage of a technology from its social, consumer diffusion to users. In fact, the idea that a technology is developed without an end user in mind runs counter to both intuitive notions of how design is accomplished and also anecdotal studies of innovation, which both suggest that the concept of the end user is always on the mind of designers. This concept harkens back to Bruno Latour's 1987 critique of the diffusion model [9], wherein Latour points out that if so many people unthinkingly accept a technology (as it is proposed in the diffusion model), it would seem as if objects themselves compelled users to work with them - but what, he asked, is this "inertia that does not depend on the action of people" that propels them? Latour states that the simple belief in diffusion does not account for the "long translations" that were necessary to bring different actors into an alliance that would, for instance, produce vaccinations or the Diesel engine. Latour's diffusion model points to the active role that users take in the development of technology as well as lends credence to the idea that creating juxtapositions of hypothetical users with technological systems is an important aspect of innovation.

I would like to extend this analysis of "translations" with the study of the history of the Internet. Recounting the story of the complex interactions between users behind the innovations that led to the Internet is similar to Latour's effort to counter the mythic, passive society that is constructed in order to support the theory of diffusion to simple users. The story of the Internet's adds to Latour's translation model the necessary substrate of the international scientific community. The TCP/IP Internet did not succeed because it was a good technological innovation that either was inevitably accepted by large groups of users based on its merits nor, as is sometimes asserted by my students, because it was enforced through the military and cultural might of the United States over other alternatives like the proposed OSI model. Basing the Internet's eventual success on the diffusion model hides the work of the many actors that added their insights to create a flexible protocol that would work in unexpected circumstances. It was the efforts of these individuals - and their ideal of communication that would function through networks that were locally administered, as would be the case in international contexts where national governments have control over communication or in business communities where networks are designed to aid the enterprise - that accounts for the Internet's success. The translational activities of these user groups, however, is not often told when one considers the history of the Internet.

Before the conference, a group of us was fortunate enough to visit The National Museum of Computing at Bletchley Park on Sunday. One of the clearest indications that the international community is essential to the history of the Internet is the museum's exhibit on Donald Davies. Donald Davies, at the National Physical Laboratory in the England, made a proposal for a "national message switching network" for commercial applications. A paper written by Davies his colleagues uses the word "packet" for the first time and describes a common-carrier network. The 
IFIP conference as well has allowed us to think about why this story matters. At the conference, Gauthier van den Hove's insight that one way to make the history of computing relevant is to utilize it as inspiration for the present. Several presenters have done the same, and it is something that I know is successful based on my own teaching of courses like Hypertext in Context at the Polytechnic Institute of NYU. I think one reason why people do not think it is useful to study the history of technology is because they think technology proceeds on its own logic, the determinist version of history that Tilly Blyth in her paper said that she wants to combat.

Learning how to tell the history of a technological advance like the Internet is essential. As a field of the humanities, history is more complicated than ascertaining the facts. Historians try to understand not only the story of what happened and determine why it happened that way, but also to understand what is at stake with the common histories that are told. As pointed out by computer historian Mike Mahoney [10], histories of computing are rhetorical devices used by developers and funders of technology to make what they are doing seem natural. It is the job of the historian, then, to make sure that the right histories are being told of the right developments. If the U.S.-centric story is the history of the Internet, a certain kind of innovation policy is implied: one must enhance the ability of national projects in partnership with the military. But what about the other history of the Internet, the one that focuses on the international community? If this story is plausible, then it implies a different kind of technology policy, one that enhances international cooperation.

\section{The Military Origin Myth}

One of the most common myths about the Internet is that it was started as a military communication project to withstand a nuclear attack. Like many myths, this reflects reality to a certain extent, even though the founders of the Internet have gone to great lengths to revise the record to state that this idea is distorted (Larry Roberts, for instance, is quoted by Andreu Veà [11] as saying that ARPANet had "nothing to do with nuclear war or survivability"). One of the technical achievements of Internet technology is the use of packets, which means that a long message is split into short units of a predetermined length. As documented by Les Earnest [12], the first time that packetized data was used in communications was for the SAGE missile defense system. SAGE was a network of computers designed to integrate twenty-two radar stations around the perimeter of the United States and it was designed to detect an aircraft carrying a heavy payload - in other words, to prevent the Soviet Union doing to the United States what the United States had done to Japan in 1945: detonating a nuclear device over its cities. SAGE was not exactly a success; it became operational in 1954 and it could track aircraft and send out intercepting fighter jets. However, with the use of ballistic missile that could carry nuclear weapons, the SAGE system was obsolete almost before it began.

Nevertheless, as documented by Paul N. Edwards [13] and others, SAGE was successful in unexpected ways. It pioneered important technologies, such as computer screens, modems, and computer memory, not to mention transmission of information in packets. This system would not have survived a nuclear attack, however, for two 
reasons. One was because it had a centralized command center, so disabling the command center would have ended the system's capabilities. The other was that the system used ordinary telephone lines for transmissions; certainly, the copper wires used for the telephone network in the 1950s could not have withstood a nuclear attack.

SAGE is visible not only in the histories of the Internet but also in mass media. Feature films such as Fail Safe (1964), Dr. Strangelove, or How I Learned to Stop Worrying and Love the Bomb (1964), and WarGames (1983) all depict computer systems that resemble SAGE. This visibility, however, hides a robust international community that existed at the time SAGE was developed. As documented by William Aspray [14], computing had already spread to fifteen countries by 1955, and surprisingly, government did not play a large role in countries outside the United States and Britain. Even in the U.S. and Britain, where government played a larger role, an equal number of computers were built by universities as were built by government. This transfer of technology would later be accomplished by commercial means, but as Aspray points out, it was initially accomplished by the mechanisms of "international science": "reports, professional journals and monographs, visits, secondment, and professional conferences" (356). This is not surprising, given the robust international scientific and technical community that had developed over centuries, and yet it is often omitted from the histories of the Internet.

The use of data packets for SAGE was not the only thing that was used in the creation of the Internet; the Internet is an example of packet-switched technology. The first large-scale test of packet switching began in 1969, when the Advanced Research Projects Agency, an agency of the Department of Defense, established a computer network for research purposes. However, there are ten years between the use of packets in SAGE and the packet-switched network known as ARPANet. How did this idea find enough advocates in these ten years so that the idea would seem obvious to the ARPA leaders that the time had come for packet-switching? The innovation of implementing a switched network was thought about in three different places in the late 1950s and early 1960s. Leonard Kleinrock's 1961 doctoral dissertation at MIT provided mathematical models to support the idea of packet switching, proving that it would work. Paul Baran, working on a military communication project for the Air Force, reported in 1964 that a switched network was the most reliable way to set up a survivable emergency communication network. Finally, Donald Davies at the National Physical Laboratory in the United Kingdom made a proposal for a "national message switching network" (225-6) for commercial applications; it was not until the next year that it was brought to Davies's attention that Baran had made a similar proposal [15]. Although some have suggested that packet-switching was developed independently by two or three different researchers, it would be more accurate to state that the research was conducted collaboratively.

The thesis that direct international cooperation was essential for the development of the Internet is supported in this period. Davies and three colleagues used the word "packet" in a paper presented by R. A. Scantlebury at the same ACM symposium in 1967 where Larry Roberts made the first public presentation of the funding priorities for the ARPA network [16]. These three individuals who made well-known testimonials for packet switching discussed the idea with the head of ARPA and convinced him to use packet-switching for his network. In preparation for developing 
the British network, Scantlebury (along with K. A. Bartlett and P. T. Wilkinson) developed a single-bit handshaking procedure for transmitting data over a serial link reliably, a protocol that was eventually adopted for the ARPANet in a modification to the IMP software in 1972. The adaptation of the "odd/even" packet acknowledgement improved network efficiency by 10 to 20 percent [17]. The international academic transfer of technology as described by [14] clearly extended to the age of the ARPANet; additionally, it is important to note that Davies and his team sought commercial applications for their network and were not interested in military research or survivability in the event of nuclear attack.

Of the three people - Kleinrock, Baran, and Davies - one of them did in fact design a network to withstand a nuclear strike. Baran's network [18] was innovative in the way that it created redundant pathways between important locations in the network. Baran imagined that telephone lines were part of the network, but also there would be microwave transmitters, FM radio transmitters, buried cables, satellite links, and other paths that the messages could take. This robust and redundant network would have survived a nuclear attack; it also would have been inordinately expensive for widespread use. The Air Force declined to pursue his plan for a defense communication network, and so the project was ended. The main legacy of this network (with regard to the Internet, anyway) is that Baran's paper inspired Donald Davies and influenced the decision of ARPA to start the widespread use of a switched network. (Interestingly, Arroyo [7] suggests that this work was not discussed with the researchers working on the Spanish network.)

The ARPAnet was the one project that was built and expanded, while Baran's project was never tested and Davies' did not get far. Although ARPANet was a defense department project, there is one definite way in which it was not a network to survive a nuclear attack: that is because it, like SAGE, used telephone lines for its connections. In this way, it is quite different from the network that was imagined by Paul Baran but never built. Nevertheless, the myth that the Internet was designed by military generals as a communication network to withstand a nuclear attack is quite pervasive, even though the Internet pioneers (including Baran) have tried hard to discredit it. This myth supports other widespread myths about the Internet. If we believe the myth that the Internet was invented to survive a nuclear attack, it is much easier to believe the myth that the Internet was started and perfected by the United States before it was sent out to other countries. This myth is very satisfying to Americans, but it is not very ethical.

The development of the Internet depended on the international community of researchers from the start. One of the three early proponents of switched networks was Donald Davies from Great Britain. The fact that he heard of the idea from the U.S., tested it out in the UK, and then came to a computer conference in the United States and talked to the leader of the ARPANet project demonstrates how these ideas were fully discussed and shared in an international atmosphere. In fact, the word we use to describe this technology - packet -makes more sense in the context of British English. Baran had called the technology message-switching, but the British influence is shown in the original ARPAnet documentation, which called the technology "packet-switching." A "packet," in British English, is a small, flat package, and this connection does more than provide evidence of the British influence on Internet technology. The idea of the helpful post office that knows how to deliver packages 
was described in Klienrock's paper. Furthermore, as pointed out by the Oxford English Dictionary [19], the packets that one receives in the mail are related to the packet boats that carried them; the first packets were standard shipping of letters between two points, such as England and Ireland. Thus, by appreciating the international community behind the early Internet technology, one can see how it was built by translating ideas from successful networks already in operation to electronic networks. This shows how users translate new ideas from concepts in other domains in order to actively support innovation.

\section{From ARPANet to Internet}

The ARPANet was publically demonstrated at the 1972 International Conference on Computer Communications, and as noted by Abbate and others, it was at this conference that the International Networking Working Group (INWG) was formed, with Vinton Cerf in charge of the group (this group would become IFIP Working Group 6.1 [20]). With the success of a national project for networking, the idea of the group was to "take the lead in creating an international network of networks" (quoted by [1]). The group quickly allied itself with the International Federation for Information Processing (IFIP), giving it "visibility and legitimacy within the international computer science community" [3]. It is, perhaps, not surprising that the international conversation around the innovation of packet switching before ARPA financed its networking project was embodied in the conception of the user as an international scientist after ARPAnet was established.

In the wake of the INWG's second meeting in 1973, Cerf edited and distributed a draft of was then called the "International Transmission Protocol" created with an international group of researchers who had experience in packet switching [20]. By 1973, Davies had an operating packet-switched network in his laboratory, and that same year, the first connections were made for the CYCLADES network in France, which "links several major computing centers" [21]. Although today one might be tempted to see these as distinct projects, it is clear that there was an active exchange of ideas among the researchers involved; furthermore, what one might see as different networks today may not have been so distinct at the time: as noted by [6], "ARPANet was not notably different to several other embryonic internetworking research projects": the UK's EPSS, CYCLADES, and the European Informatics Network (25). It is clear that the ARPANet, at this point in the story, was not the only packetswitched network in the world.

What is more, the ARPANet was not the Internet: The ARPANet would have to adapt its implementation of packet-switching in order to connect heterogeneous networks, and this shows evidence of additional international collaboration. Cerf and Robert Kahn started working on what they called the "Internetworking" project at this time: how could someone send a message between different networks? They presented their ideas to a 1973 meeting of the INWG at the University of Sussex [1]. In one of their first published papers on this topic, they note that the design goal is to establish protocols that eliminate the problems when "dissimilar networks are interconnected." This 1974 paper [22] acknowledges that the "early discussions of international network protocols" were helpful, and mentions Scantlebury and Davies 
by name. In addition, they thank and cite the work of Louis Pouzin, the French researcher behind CYCLADES. In creating a protocol that could easily work between networks, it was clear that the restrictions and capabilities for each network were different because they had different physical properties. To accommodate this, one of the key ideas in TCP/IP is that each packet needs a wrapper. Packets that originate on different networks might be set up differently, and this wrapper would help the networks carrying the message work with it, even though they could not know what the contents were. Cerf and Kahn acknowledge that they borrowed the solution from the French computer science community. CYCLADES had a slightly different implementation, and this difference resulted in an innovation that was applied directly to the TCP/IP protocol. The name CYCLADES comes from a group of Greek islands, and this name was used for the network because the researchers wanted to inspire islands of computing. In other words, they thought about creating a mixed networking environment much like Cerf and Kahn were trying to make. Thus they borrowed the idea of the datagram created in this heterogeneous environment and imported it into their design.

With the switchover to TCP/IP in 1983, once can say that the Internet had truly arrived: after all, the " $i$ " in IP stands for internetworking. The power of TCP/IP was demonstrated many times in the expansion of the Internet. This is especially shown in the international community, where different kinds of computers and different kinds of national networks would have prevented a less flexible network from working. An excellent example of this was China's first connection to the TCP/IP network. There was no way that China could be directly connected to the U.S. Internet during the Cold War; the United States had strict laws about exporting technology to communist countries at this time. However, the U.S. was friendly to its partners. As part of the networking project known as CSNET, the U.S. made several international connections, including one to West Germany.

CSNET was established from 1979-1982 at the University of Wisconsin by Lawrence Landweber, who noted that there were 120 computer science departments at the time, even though only 25\% (15 of 61) ARPANet sites were at universities [23]. The network that emerged from funding by the National Science Foundation had three tiers: a top tier of full ARPANet connections, a second tier that send TCP/IP packets over commercial X.25 lines so that researchers could use services like mail, telnet and FTP, and an e-mail only service. Along with setting up the service, Landweber held a series of meetings that came to be known as Larry's Networkshops, which "formed an important way, along with BITNET, to spread the Internet overseas" [24]. The first meeting in 1982 was held in London, where German researchers described plans for Deutsche Forschungsnetz (DFN), active projects in Norway and Sweden were described, the U.K. described the Coloured Book, and CERN described an early version of HEPnet. At the 1983 networkshop, Landweber announced that CSNET would go international, and the next year, Israel became the first connection outside the U.S. Connections to Korea, Australia, Canada, France, Germany, and Japan followed.

Landweber's effort to support the story of international connections shows how today's ubiquity of TCP/IP was not inevitable. As told by Andrew L. Russell [25], at the time TCP/IP was thought of as a temporary solution to international networking that would later be supplanted by protocols under development by the International 
Organization for Standardization known as Open Systems Interconnection (OSI). Remarkably, Vinton Cerf had resigned as chair of INWG after becoming "discouraged by his international adventures in standards making" (40). By 1985, the U.S. Department of Defense recommended a transition away from TCP/IP and in 1988, Russell writes, the U.S. Department of Commerce mandated that all government computers use OSI by 1990. Russell [26] has characterized this conflict as a "protracted international struggle" (56), even though today the discussion is largely forgotten. It is clear, however, that the international community involved in networking research resented a top-down approach to generating standards, an attitude that is nicely summarized in David Clark's 1992 dictum "We reject: kings, presidents, and voting. We believe in: rough consensus and running code" [27]. By 1992, Clark was able to refer to the defeat of OSI as an example of how the grassroots commitment to Internet development had provided the world with a working network, but the ability of the international community to successfully resist top-down demands for standards was far from certain.

An example of how "running code" became the default standard for the Internet is seen in the story of how China was connected to the international Internet. West Germany had a robust computer industry, and through a contract with the World Bank, Siemens Corporation had supplied computers to China and helped connect computer science researchers in both companies. Werner Zorn, who was responsible for organizing German visitors to China, was dismayed that he could not send e-mail to his Chinese colleagues: "A turnaround time of 14 days is even considered fast if one does not want to resort to the extortionately expensive telephone or telex, which are not available everywhere" [28]. He then tried to set up a modem connection between the two countries, but the phone lines in China were not reliable enough to sponsor communication. He ended up using the CSNET software - modified to the Chinese setup of Siemens computers, of course - to send TCP/IP packets through a satellite connection that China had with Italy, and then through the European networks to Zorn's university computer [29]. Finally, his colleagues could exchange email messages with the rest of the world. The development of TCP/IP utilized a conception of a user who was an international scientist managing a local network, and this definition of a user enabled Zorn to connect his Chinese counterparts to the Internet in spite of Cold War prohibitions about the export of technology.

In stories like this, one sees how it was not the inevitable progress of a military objective that led to the widespread adoption of the Internet, but instead the value of translational work of individuals who sought to enhance their access to the value of the versatile networking protocols that were developed with the help of the international community, and how the desire to connect different networks in different countries helped TCP/IP to become the dominant networking standard. If we want to be unfriendly to the United States, we could say that the U.S. researchers stole these ideas from their foreign colleagues and used them for their own work. But the reality is that this sharing has a long tradition in the history of science, and the Internet is no different than any other scientific endeavor. The idea of sharing ideas in an international forum and then making the ideas work in one's home environment is one of the established principles of the scientific community. The myth that the Internet is a U.S. invention, then, hides how it was developed and tested with the conception of an ideal user who was a member of an international group of scholars. 


\section{Conclusion}

Dispelling the myth that the United States created the Internet in isolation is crucial if one wants to recreate the environment that brought about the Internet in the first place. If the military made the Internet, then it would seem that one needs to give more money to the military to continue this kind of innovation. If the United States developed the Internet on its own, then there is no need to foster international cooperation. If the Internet was made according to a plan, then one needs to keep research tied to a clearly specified timeline with clear goals and deadlines. However, the way to invent the Internet (or the next generation of Internet technologies) is quite opposite to what these myths would lead us to believe. The technologies behind the Internet were invigorated by the international community, not by the United States in isolation. In order to create something like the Internet, then, it is necessary to foster an environment for international exchange and cooperation, not maintain proprietary secrets. The historian has an important role to play in making sure this environment is available. If we allow the myths of the Internet to perpetuate, we give support to misguided projects that will not be able to create the innovations of tomorrow.

Clearly, the popular history of the Internet needs to be revised. Sponsoring insular, high-tech research projects is not the way to create the next generation of Internet technologies. The story of the academic, international Internet is one that encourages us to foster heterogeneous connections. It is the role of the historian to remind policy makers, educators, and the users of the Internet that it was not planned from the start. It was hacked together by users of different projects to help them do the kind of work they found most valuable. In order to make the history of computing relevant, it is the task of the historian to show how the cosmopolitan tradition of science is responsible for the Internet we know today, and in order to foster this kind of innovation in the future, this community must be supported.

\section{References}

1. Hafner, K., Lyon, M.: Where Wizards Stay Up Late: The Origins of the Internet. Touchstone, New York (1996)

2. Segaller, S.: Nerds 2.0.1: A Brief History of the Internet. TV Books, New York (1999)

3. Abbate, J.: Inventing the Internet. MIT Press, Cambridge (1999)

4. Mueller, M.L.: Ruling the Root: Internet Governance and the Taming of Cyberspace. MIT Press, Cambridge (2002)

5. Campbell-Kelly, M., Garcia-Swartz, D.D.: The History of the Internet: The Missing Narratives. J. of Information Technology 28, 18-33 (2013)

6. Kim, B.K.: Internationalizing the Internet: the Co-evolution of Influence and Technology. Edward Elgar Publishing, Northampton (2005)

7. Arroyo, L.: The First Worldwide Public Packet Switching Network. In: A Century of Broadcasting: Proceedings of the Second Region 8 IEEE Conference on the History of Communications. IEEE, Piscataway (2010)

8. Oudshoorn, N., Pinch, T. (eds.): How Users Matter: The Co-Construction of Users and Technology. MIT Press, Cambridge (2005)

9. Latour, B.: Science in Action: How to Follow Scientists and Engineers through Society. Harvard University Press, Cambridge (1987) 
10. Mahoney, M.S.: Histories of Computing. MIT Press, Cambridge (2011) Haigh, T. (ed.)

11. Veà, A.: The Unknown History of the Internet: Engineering the Worldwide WiWiW Project. In: A Century of Broadcasting: Proceedings of the Second Region 8 IEEE Conference on the History of Communications. IEEE, Piscataway (2010)

12. Earnest, L.: Internet Creation Myths, http://www.stanford.edu/ learnest/ net.htm

13. Edwards, P.N.: The Closed World: Computers and the Politics of Discourse in Cold War America. MIT Press, Cambridge (1997)

14. Aspray, W.: International Diffusion of Computer Technology, 1945-1955. Annals of the History of Computing 8(4), 351-360 (1986)

15. Campbell-Kelly, M.: Data Communications at the National Physical Laboratory. Annals of the History of Computing 9(3/4), 221-247 (1988)

16. Davies, D.W., Bartlett, K.A., Scantlebury, R.A., Wilkinson, P.T.: A Digital Communication Network for Computers Giving Rapid Response at Remote Terminals. In: Proceedings of the First ACM Symposium on Operating System Principles, SOSP 1967, pp. 2.1-2.17. ACM, New York (1967)

17. McQuillan, J.M., Crowther, W.R., Cosell, B.P., Walden, D.C., Heart, F.E.: Improvements in the Design and Performance of the ARPA Network. In: AFIPS Fall Joint Computing Conference, vol. 2, pp. 741-754 (1972)

18. Baran, P.: On Distributed Communications, 1: Introduction to Distributed Communications Networks. RAND Corporation, Santa Monica (1964)

19. Oxford University Press: Packet, n. and adj. OED, http://www. oed.com/view/ Entry/135850 (accessed August 11, 2013)

20. McKenzie, A.: INWG and the Conception of the Internet: An Eyewitness Account. Annals of the History of Computing 33(1), 66-71 (2011)

21. Roberts, L.G.: The Evolution of Packet Switching. Proceedings of the IEEE 66(11), 1307-1313 (1978)

22. Cerf, V.G., Kahn, R.E.: A Protocol for Packet Network Intercommunication. IEEE Transactions of Communication 22(5), 637-648 (1974)

23. Comer, D.: The Computer Science Research Network CSNET: A History and Status Report. Communications of the ACM 26(10), 747-753 (1983)

24. Malamud, C.: Exploring the Internet: A Technical Travelogue. Prentice Hall, Englewood Cliffs (1993)

25. Russell, A.L.: The Internet that Wasn't. Spectrum 50(8), 39-43 (2013)

26. Russell, A.L.: "Rough Consensus and Running Code" and the Internet-OSI Standards War. Annals of the History of Computing 28(3), 48-61 (2006)

27. Clark, D.: A Cloudy Crystal Ball: Visions of the Future. In: Proceedings of the TwentyFourth Internet Engineering Task Force, pp. 539-543. Corporation for National Research Initiatives, Reston (1992)

28. Zorn, W.: How China was Connected to the International Computer Networks. The Amateur Computerist Newsletter 15(2), 36-49 (2007)

29. Zorn, W.: China's CSNET Connection 1987 - origin of the China Academic Network CANET. Asia Internet History Project, https://sites.google.com/site/ internethistoryasia/book1/personal-essay-werner-zorn (accessed April 21, 2013) 